\title{
Cayenne Pepper Cataplasm "Munari" reduces pain and improves mobility in patients with non-specific chronic low back pain
} \author{
Kern $^{5}$, Wolfgang Hüebl ${ }^{8}$ and Helmut Kern ${ }^{4,5 *}$ \\ ${ }^{1}$ Department of Biomedical Sciences, Padova, Italy \\ ${ }^{2}$ University of Primorska, Faculty of Health Sciences, Koper, Slovenia \\ ${ }^{3}$ S2P, Science to Practice Ltd., Laboratory for Motor Control and Motor Behaviour, Ljubljana, Slovenia \\ ${ }^{4}$ Ludwig Boltzmann Institute of Rehabilitation Research, St Pölten, Austria \\ ${ }^{5}$ Institute of Physical Medicine and Rehabilitation, Prim. Dr. H Kern GmbH, Amstetten, Austria \\ ${ }^{6}$ Comenius University, Institute for Human Performance, Bratislava, Slovakia \\ ${ }^{7}$ Comenius University, Department of Sport Kinanthropology, Bratislava, Slovakia \\ ${ }^{8}$ Department of Laboratory Medicine, Wilhelminenspital, Vienna, Austria
}

Sandra Zampieri ${ }^{1}$, Nejc Sarabon ${ }^{2,3}$, Stefan Löfler ${ }^{4}$, Christian Hofer ${ }^{4}$, Sascha Sajer ${ }^{4,5}$, Felix Kabas ${ }^{4}$, Jan Cvecka ${ }^{6}$, Milan Sedliak $^{7}$, Mathias

\begin{abstract}
Objective: To test the cumulative effects of repeated application of Cayenne Pepper Cataplasm "Munari" alone (MUN) or in combination (COMB) with neuromuscular electrical stimulation (NMES) and massage on non-specific chronic low back pain (LBP) in terms of safety, pain relief, function and mobility.

Design: Before and after intervention trial. Adults suffering from non-specific chronic LBP were treated with "Munari", ten times within 3 weeks. In COMB, following "Munari", 15 minutes of massage and 15 minutes of NMSE were performed. Blood pressure, circulating levels of acute phase proteins (ESR, CRP, CK, albumin), cortisol and inflammatory biomarkers (TNF- $\alpha$, IL-6, sp-selectin) were assessed before the $1^{\text {st }}$ and after the $10^{\text {th }}$ treatment. At the same time points, measurements included VAS questionnaire, function and mobility tests (ODI, Schober), and serum levels of miRNAs involved in inflammation, pain signalling, and muscle homeostasis.

Results: In MUN, blood pressure and pain were significantly reduced. In COMB, function and mobility test's scores significantly improved. In both groups, circulating levels of acute phase response or inflammatory biomarkers didn't increase, and miRNAs regulating pain signaling were significantly modulated.

Conclusions: Repeated Munari applications are safe and effective for the treatment of non-specific chronic LBP, in particular when combined with NMES and massage.
\end{abstract}

\section{Introduction}

Low back pain (LBP) is defined as pain between the costal margins and the inferior gluteal folds, with or without leg pain [1]. In the great majority of cases (up to 90\%) it is non-specific being not related to systemic conditions, fractures, or direct trauma. Only in $10 \%$ of cases it is related to specific pathologies. Chronic LBP is by definition - LBP with a duration of at least three months.

Low cost treatments such as natural medications are often used to treat painful or aching areas in the case of acute or chronic LBP [2-5]. Many remedies consist of topical herbal applications containing rubefacient substances (e.g. Cayenne pepper, CP) or wraps that generates local heat by a chemical reaction of activated carbon and iron powder. The most abundant alkaloid presents in $\mathrm{CP}$ is Capsaicin, a highly selective agonist of the Transient Receptor Potential Vanilloid 1 (TRPV1) [6]. This cathion channel permeable to calcium $\left(\mathrm{Ca}^{2+}\right)$ is localized on cutaneous and muscle nociceptive sensory nerves that carry afferent pain [6].
Binding of Capsaicin to TRPV1 increases intracellular $\mathrm{Ca}^{2+}$ concentration, activating $\mathrm{Ca}^{2+}$-dependent proteases and directly inhibits the mitochondrial electron transport chain, thus inducing mitochondrial dysfunction $[7,8]$. These two mechanisms result in defunctionalisation of the nerve fibres and are responsible for pain reduction and long-lasting desensitization of the treated area [9].

In 1909 Dr. Giuseppe "Munari” described a preparation composed of rubefacient substances which generated vasodilation and increase in blood circulation in the treated areas. This method was used to treat pain in different parts of the musculoskeletal system and became famous in Italy and the neighbouring countries. In Austria a similar application

${ }^{\star}$ Correspondence to: Helmut Kern, Ludwig Boltzmann Institute for Rehabilitation Research, Reizenpfenninggasse 11140 Vienna, Austria, E-mail: helmut@kern-reha.at

Key words: Low back pain, capsaicin, trpv1 receptor, massage, nmse

Received: June 12, 2019; Accepted: June 21, 2019; Published: June 28, 2019 
consisting of $\mathrm{CP}$ and Kaolin powder (the so called "Munari"), containing low dose Capsaicin, is still commonly used to treat LBP and other musculoskeletal dysfunctions [4]. Although the treatment is frequently applied and generally well tolerated, only very few scientific papers were published regarding its therapeutic effect $[4,10]$. In part, this is because the treatment has a long history in Physical Medicine dating back to the time when medications were developed on empiric observations [11].

However, the acquirement of new methods does not necessarily mean that the "historical" treatments have no therapeutic effect. Unfortunately, a study financed by the Federation of the Austrian Social Insurances Entities and conducted by Enthaler et al. [12]. concludes that missing evidence for low dose $\mathrm{CP}$ application equals no efficacy of the treatment. Only possible side effects are mentioned, without proofs. No studies and no data about potential risks and secondary effects of this kind of treatment are available. So far scientific studies concerning capsaicin treatment in LBP only investigated high dose CP patches (Qutenza ${ }^{\circledR}$ containing 8\% Capsaicin) $[7,8,13,14]$.

Up to now, no circulating biomarkers can be considered specific for LBP or can predict predisposition of disease or disease progression. Besides peptides and proteins, microRNAs (miRNAs) are of increasing interest as signalling molecules or injury biomarkers. MiRNAs are small non-coding RNAs that negatively regulate gene expression at post transcriptional level [15] playing a role in a wide range of biological and pathological processes. Expression changes of miRNAs profile has been observed also in a variety of painful conditions [16] but have currently been scarcely studied in pain physiology. Because of their size, abundance, tissue specificity, and relative stability in plasma, miRNAs show promise as unique accessible biomarkers to monitor tissue injury, or a response to therapeutic modalities.

In our previous study, we have shown that one time 20 minutes application of low dose CP (containing $0.09 \%$ of Capsaicin) has positive effects on selected functional and mobility parameters, without side effects on circulating levels of selected inflammatory biomarkers [10].

Intention of the present work is to examine the effects of repeated low dose CP cataplasm (CPC) as a monotherapy (MUN) or in combination with neuromuscular electrical stimulation (NMES) and massage (COMB) on chronic non-specific LBP. Using blood samples collected before the $1^{\text {st }}$ and after the $10^{\text {th }}$ treatment, we analysed the expression of circulating levels of a selection of biomarkers, including miRNAs, involved in inflammation, pain and Capsaicin related mechanisms of action, in association with pain, mobility and functional parameters.

\section{Materials and methods}

\section{Participants}

Male patients suffering from chronic non-specific LBP, were assigned in alternating order of appearance into two groups: "Munari" group (MUN, $\mathrm{n}=10$, mean age $65.3 \pm 7.3 \mathrm{yrs}$, BMI $27.6 \pm 4.1 \mathrm{~kg} / \mathrm{m}^{2}$ ) and combination therapy group $(\mathrm{COMB}, \mathrm{n}=10$, mean age $57.7 \pm 5.3$ yrs, BMI $27.7 \pm 2.7 \mathrm{~kg} / \mathrm{m}^{2}$ ). All subjects volunteered to the study and gave their written informed consent. Approval of the responsible ethical committee was obtained at Comenius University, Sports Faculty of Bratislava (nr. 2017LBI01) and clinical trial registration under clinicaltrials.gov, number NCT03440125. Inclusion criteria for all subjects were: male, age of 18-75 years having a BMI below $35 \mathrm{~kg} / \mathrm{m}^{2}$ and suffering from non-specific chronic LBP (for at least 3 months).
Sensibility disorders (e.g. due to neurological disease), injuries, open wounds or rash in intervention area, known hypersensitivity against the applied substances, HIV, Hepatitis $\mathrm{C}$ and other due blood communicable infectious diseases were exclusion criteria for all subjects. Recruitment, measurements and therapies were performed at Comenius University, Sports Faculty, Bratislava (Slovakia).

\section{Experimental design}

Each subject in both groups, MUN and COMB, underwent 10 times application of 5\% CPC patches (0.09\% Capsaicin content) for 20 min within 3 weeks as described [10]. In COMB group a combination of therapies was performed afterwards starting with i) 15 min classical back massage, and finally with ii) 15 min NMES on the lower back with Stimulette rx device (Dr. Schuhfried Medizintechnik GmbH, Van Swieten-Gasse 10, A-1090 Wien, Austria). A frequency-modulated stimulation was applied using the following parameters: biphasic triangular impulses with $0.8 \mathrm{~ms}$ pulse width and a 1-second inter-burst interval, which is modulated from $3.3 \mathrm{~Hz}$ to $32.9 \mathrm{~Hz}$ every 3 seconds. Physiotherapists delivered Munari applications and combination therapies.

\section{Blood sampling}

Blood samples from non-fasted subjects were collected before the $1^{\text {st }}$ and after the $10^{\text {th }}$ treatment, $0.5 \mathrm{hrs}$ and $48 \mathrm{hrs}$ post the $10^{\text {th }}$, to evaluate acute and long-term effects.

Venous blood samples $(7.5 \mathrm{ml})$ were collected in a serum separator collection tube for serum isolation and in EDTA tubes for plasma separation by centrifugation carried out at $3000 \mathrm{rpm}$ for $10 \mathrm{~min}$.

\section{Measurement procedures and clinical tests}

Blood pressure was measured using an automatic barometer with the cuff placed around the upper arm. Two measurements were made, one before the $1^{\text {st }}$ and one at the end of the $10^{\text {th }}$ treatment. The whole measurement procedure was run on both arms according to the manufacturer's instructions for use.

Questionnaires (VAS) and clinical tests (ODI and Schober) were performed before the $1^{\text {st }}$ and $48 \mathrm{hrs}$ after the $10^{\text {th }}$ treatment after blood samples were taken, in order to avoid clinical testing performance on possible blood parameter changes.

Visual analog scale (VAS) with a range from 0-100 $\mathrm{mm}$ was used to evaluate average pain in activities of daily living at rest (VASr) and during activity (VASa) in the last $48 \mathrm{hrs}$ prior to the assessments. Value 0 represents absence of pain and value 100 represents worst possible pain.

Oswestry Disability Index (ODI), a standard questionnaire to measure the subject's permanent functional disability for LBP, was used [17]. It contains ten topics, scored on a scale of 0 - 5. All scores were summed up and then multiplied by two to obtain the index. Zero corresponds to no disability and 100 is the maximum disability possible.

Schober's test was used to measure the ability to flex the lower back. Subject assumes an upright position without clothes (except underwear) [18]. The examiner then locates spinous process S1, marks it and makes a second mark $10 \mathrm{~cm}$ cranially. The patient is asked to perform three maximal trunk flexions, with knees straight. The greatest distance between these two marks out of three repetitions is taken for further statistical analysis. 


\section{Serological assessments}

\section{Clinical chemistry and laboratory parameters}

The Erythrocyte Sedimentation Rate (ESR) were calculated with an Alifax THL sedimentation analyser. C-reactive protein (CRP), albumin, and creatine kinase (CK) were analysed in serum samples on a Siemens Dimension Vista 1500 multiparametric analyser, cortisol was analysed on a Siemens Centaur XP Immunoassay System. Whole procedures were run according to the manufacturer`s instructions for use.

\section{Inflammatory biomarkers}

Tumor Necrosis Factor alpha (TNF- $\alpha$ ), Interleukin 6 (IL-6), and soluble platelet selectin (sP-selectin), were measured in serum samples by ELISA. All kits were purchased from R\&D Systems (Human TNF- $\alpha$ Immunoassay HSTA00D, Human IL-6 Immunoassay HS600B, and Human sP-selectin/CD62P Immunoassay BBE6). All procedures were run according to the manufacturer's instructions for use. To avoid inter-assay variations, all samples were analysed in duplicates with the same kit on the same day.

\section{miRNA profile}

We selected a panel of miRNAs based on prior evidences for a role in cellular processes modulated by inflammation and Capsaicin topical treatment. Specifically, we chose miRNAs regulating TRPV1 transcription (miR-199a, and miR-199b) [19,20], those mediators of inflammation (miR-155, miR-21) [21,22], endothelial cell damage (miR-126) [23] mitochondrial $\mathrm{Ca}^{2+}$ uptake (miR-25) [24] and skeletal muscle homeostasis (miR-1, miR-133, and miR-206) [25,26]. A geNorm-like algorithm was applied to our data in order to select the three most appropriate miRNAs for normalization. The algorithm identified hsa-miR-146a-5p, hsa-mir-23a-3p, hsa-miR-191-5p to normalize the data in our experiment. hsa-mir-451a and hsa-mir486-5p were also included in the panel as hemolysis markers to detect blood cell contamination in pure plasma samples. MiRNAs detection was performed using the Multiplex Circulating miRNA Assay using Firefly ${ }^{\mathrm{TM}}$ particle technology, a new technique that enables the profiling of miRNAs of choice directly from plasma, with no need for RNA purification with readout on standard flow cytometers [27]. Raw data was analysed using the FirePlex Analysis Workbench. Fold change differential expression for the gel-particle hybridization flow cytometry experiments was calculated as the ratio between normalized expression of each miRNAs after the last treatment ( $0.5 \mathrm{hrs}$ and $48 \mathrm{hrs}$ post) and normalized expression of each miRNA before the treatment.

\section{Statistical analyses}

Statistical analysis (using SPSS 20.0 software, SPSS Inc., Chicago, USA) was performed as follows. Descriptive statistics were calculated for all parameters and reported as mean \pm standard error (SEM). Shapiro-Wilk test was used to assess the normal distribution of data. For evaluating effects of different treatment modalities at three points in time, 2-ANOVA (time $(3) \times$ condition $(2)$ ) was performed. For parameters that were assessed only at two points in time, this was reduced to time $(2) \times$ condition (2). Additionally, repeated measures of ANOVA or paired t-tests were performed to assess therapy effects separately within each group. Effect size was assessed with $\eta^{2}$ test and interpreted as small $(\sim 0,01)$, medium $(\sim 0,06)$ or large $(\sim 0,26)$. Pairwise comparisons within 2-way ANOVA were calculated with Bonferroni corrected t-tests.

\section{Results}

All enrolled subjects $(n=20)$ completed the study performing all measurement procedures and clinical tests.

\section{Repeated "Munari" applications reduce blood pressure}

One of the negative systemic side effects of a single treatment with Qutenza ${ }^{\circledR}$ patches (8\% Capsaicin content) is the increased blood pressure [28]. In our study, after 10 times "Munari" applications $(0.09 \%$ Capsaicin content), a significant reduction of the systolic (139.40 \pm 4.37 vs. $132.60 \pm 4.11 \mathrm{~mm} \mathrm{Hg}, \mathrm{p}=0.05)$ and diastolic $(86.00 \pm 4.62$ vs. $81.10 \pm$ $3.63 \mathrm{~mm} \mathrm{Hg}, \mathrm{p}=0.03$ ) blood pressures was observed in MUN group. No significant changes in blood pressure either systolic (pre 127.25 \pm 3.56 vs. post $127.75 \pm 2.61 \mathrm{~mm} \mathrm{Hg}$ ) and diastolic (pre $86.00 \pm 2.38 v s$. post $83.17 \pm 1.46 \mathrm{~mm} \mathrm{Hg}$ ) were observed in COMB group.

\section{Circulating levels of acute phase response and inflammatory biomarkers are not increased after repeated "Munari" treatments}

Qutenza ${ }^{\circledR}$ patche application induces local side reactions such as redness, itching, burning and swelling, resulting from the evoked inflammatory response at the site of application. "Munari" treatment, consisting of a low dose Capsaicin, induces some of these signs of inflammation appearing immediately after the application, but resolving in a short period of time, within a few hours.

The activation of the inflammatory response at the site of application does not result in significant or long-lasting changes of the acute phase response biomarkers (CRP, ESR, Albumin, and CK, Table 1), or of the pro-inflammatory cytokine TNF- $\alpha$ (Table 1). In MUN group, we observed a tendency for increased values of antiinflammatory cytokine IL-6 in response to the treatment (Table 1). In both groups, a significant decrease of biomarkers for neurogenic inflammatory response (Cortisol, and sP-selectin) was observed (Table 1). Although the changes of Cortisol levels were assessed within the normal range, those of sP-selectin were above the reference values at each point in time.

\section{Repeated "Munari" treatment is effective in reducing pain and improving mobility}

The most common clinical experience is that chronic LBP patients that are conditioned by pain, often display decreased mobility of the hips (limited lumbar tilt during trunk bending and lifting) and overflexion of the thoracic and lumbar level of the spine (trunk). After a classical Physical Rehabilitation therapy, in most of the treated patients, clinical examination shows decreased muscle tension and sensitivity to pressure, together with pain reduction and improved mobility.

Just like in our clinical experience, in our groups of subjects treated with repeated application of low dose $\mathrm{CP}$, a significant reduction of pain and significant improvement of the disability test scores were observed (Table 2). Treated subjects described less pain after the treatment, as assessed by decreased scores of VAS questionnaires. Only the MUN group showed a significant decrease in pain during activity (Table 2).

Significant reduction of the scores assessing permanent functional disability (ODI, Table 2) and functional mobility of the trunk (Schober test, Table 2), were detected in COMB group. In MUN group, the effects on mobility were milder, since mobility test scores are unchanged, and no significant reduction of ODI test scores was detected, even though a clear trend of decrease was observed (Table 2). 
Table 1. Serum levels of tested biomarkers before the $1^{\text {st }}$ and after the $10^{\text {th }}$ treatment. CRP, ESR, Albumin, CK and Cortisol reference ranges were included in each package insert instructions TNF-a, IL-6 and sP-selectin reference values correspond with the mean serum concentration determined in the laboratory, testing sera of healthy people of the same age. Values are expressed as mean \pm SEM, those significant are reported in bold; ${ }^{\$}$ Post hoc $\mathrm{t}$ test $0.029 \mathrm{vs} .0 .5 \mathrm{hrs}$ post $10^{\text {th }} ; §$ Post hoc $\mathrm{t}$ test 0.04 vs. pre ${ }^{\text {st }}$; ${ }^{\circ}$ Post hoc $\mathrm{t}$ test $0.002 \mathrm{vs}$. pre 1 st; $*$ Post hoc $\mathrm{t}$ test $0.006 \mathrm{vs}$. 0.5 hrs post $10^{\text {th }} ;{ }^{\&}$ Post hoc t test 0.017 vs. pre $1^{\text {st }}$.

\begin{tabular}{|c|c|c|c|c|c|}
\hline MUN & Reference values & Pre $1^{\text {st }}$ & 0.5 hrs Post $10^{\text {th }}$ & 48 hrs Post $10^{\text {th }}$ & RANOVA \\
\hline $\mathrm{CRP}(\mathrm{mg} / \mathrm{ml})$ & $0-5$ & $3.8 \pm 0.9$ & $3.0 \pm 0.1$ & $3.15 \pm 0.2$ & 0.438 \\
\hline ESR $1 \mathrm{hr}(\mathrm{mm})$ & $0-8$ & $8.0 \pm 6.4$ & n.d. & $9.0 \pm 8.0$ & 0.362 \\
\hline Albumin $(\mathrm{g} / \mathrm{L})$ & $35-52$ & $39.5 \pm 0.9$ & $39.9 \pm 1.3$ & $39.6 \pm 1.3$ & 0.941 \\
\hline CK (U/L) & $0-190$ & $123.1 \pm 14.4$ & $132.6 \pm 24.6$ & $117.1 \pm 13.7$ & 0.957 \\
\hline Cortisol $(\mu \mathrm{g} / \mathrm{dl})$ & $4.3-22.4$ & $12.2 \pm 1.7$ & $8.4 \pm 0.8$ & $11.1 \pm 0.8^{\varsigma}$ & 0.024 \\
\hline TNF-a (pg/ml) & 1.76 & $1.2 \pm 0.2$ & $1.2 \pm 0.2$ & $1.2 \pm 0.2$ & 0.962 \\
\hline IL-6 (pg/ml) & 1.26 & $1.7 \pm 0.3$ & $1.9 \pm 0.4$ & $2.0 \pm 0.6$ & 0.871 \\
\hline sP-selectin (ng/ml) & 41.62 & $45.2 \pm 5.5$ & $38.3 \pm 13.7^{\S}$ & $41.26 \pm 4.8$ & 0.03 \\
\hline \multicolumn{6}{|l|}{ COMB } \\
\hline $\mathrm{CRP}(\mathrm{mg} / \mathrm{ml})$ & $0-5$ & $3.0 \pm 0.1$ & $2.9 \pm 0.0$ & $2.9 \pm 0.0$ & 0.429 \\
\hline ESR $1 \mathrm{hr}(\mathrm{mm})$ & $0-8$ & $6.0 \pm 5.6$ & n.d. & $4.0 \pm 2.9$ & 0.393 \\
\hline Albumin $(\mathrm{g} / \mathrm{L})$ & $35-52$ & $40.8 \pm 0.7$ & $39.9 \pm 0.5$ & $40.2 \pm 0.6$ & 0.542 \\
\hline CK (U/L) & $0-190$ & $138.6 \pm 14.6$ & $132.8 \pm 15.1$ & $125.8 \pm 13.3$ & 0.313 \\
\hline Cortisol $(\mu \mathrm{g} / \mathrm{dl})$ & $4.3-22.4$ & $11.9 \pm 0.8$ & $9.4 \pm 0.9^{\circ}$ & $10.8 \pm 1.5^{\star}$ & 0.009 \\
\hline TNF-a (pg/ml) & 1.76 & $0.8 \pm 0.1$ & $0.8 \pm 0.1$ & $0.8 \pm 0.1$ & 0.344 \\
\hline IL-6 (pg/ml) & 1.26 & $1.4 \pm 0.2$ & $1.3 \pm 0.2$ & $1.5 \pm 0.3$ & 0.957 \\
\hline sP-selectin (ng/ml) & 41.62 & $60.8 \pm 4.5$ & $50.5 \pm 2.3^{\&}$ & $58.1 \pm 5.6$ & 0.538 \\
\hline
\end{tabular}

Table 2. Questionnaires and functional tests. Values are expressed as mean $\pm \mathrm{SEM}$; VASr $=$ Visual analog scale at rest; VASa $=$ Visual analog scale during activity; ODI $=$ Oswestry Disability Index; significant values in bold.

\begin{tabular}{|c|c|c|c|c|c|}
\hline & & VASr & VASa & ODI & Schober \\
\hline \multirow{3}{*}{ ANOVA (Interaction) } & $\mathrm{F}$ & 5.147 & 9.148 & 2.158 & 0.554 \\
\hline & $\mathrm{p}$ & 0.036 & 0.007 & 0.157 & 0.466 \\
\hline & $\eta^{2}$ & 0.222 & 0.337 & 0.108 & 0.030 \\
\hline \multirow{3}{*}{ MUN } & Pre $1^{\text {st }}$ & $2.76 \pm 1.69$ & $3.74 \pm 2.16$ & $13.0 \pm 4.6$ & $14.6 \pm 1.2$ \\
\hline & Post $10^{\text {th }}$ & $1.35 \pm 1.44$ & $1.83 \pm 2.23$ & $10.1 \pm 5.8$ & $14.7 \pm 1.3$ \\
\hline & $P$ value & 0.10 & $<0.05$ & 0.10 & 0.80 \\
\hline \multirow{3}{*}{ СОМв } & Pre $1^{\text {st }}$ & $1.94 \pm 1.94$ & $3.37 \pm 2.41$ & $19.4 \pm 9.6$ & $14.9 \pm 0.61$ \\
\hline & Post $10^{\text {th }}$ & $1.17 \pm 1.48$ & $1.78 \pm 2.36$ & $12.2 \pm 7.8$ & $14.7 \pm 0.5$ \\
\hline & $P$ value & 0.20 & 0.13 & $<0.05$ & $<0.05$ \\
\hline
\end{tabular}

2-way ANOVA revealed significant time $\times$ group interaction for VAS scores, both at rest $\left(\mathrm{F}=5.147 ; \mathrm{p}=0.036 ; \eta^{2}=0.222\right)$ and during movement $\left(\mathrm{F}=9.148 ; \mathrm{p}=0.007 ; \eta^{2}=0.337\right)$, meaning that VAS scores changed differently in the two groups between pre and post-10 time points at a statistically significant value.

Circulating levels of miRNAs regulating inflammatory response are not significantly modulated by repeated "Munari" treatment

In consistence with the detected inflammatory protein profiles, circulating levels of those miRNAs coordinating multiple signaling cascades related to inflammatory pathways were not modulated significantly. In all treated subjects, plasma profiles of miR-155, miR21 and miR-126 regulating immune-inflammatory response and angiogenesis 21-23 were unchanged.

In our analysis, we tested for miR-451 and miR-486-5p as hemolysis biomarkers as described. We did not find significantly increased values of miR-451 and miR-486-5p, indicating that no red blood cell contamination was present in our samples. Beside this aspect, since miR-451 has a physiological role in regulating the transcription of proinflammatory cytokines TNF- $\alpha$ and IL- 6 , we considered its serum levels for analysis. Indeed, in COMB group, transient changes of miR-451 were observed: its serum levels increased $0.5 \mathrm{hrs}$ after the last treatment and significantly decreased at $48 \mathrm{hrs}$, almost returning to the baseline levels (pre $1^{\text {st }}$ ).
Muscle specific miR-1 and miR-206 serum profiles are not affected by repeated "Munari" treatment

Decreased muscle tension was observed in LPB patients after the treatment. In order to verify whether skeletal muscle adaptation is related to tissue regeneration, we also tested for the expression of those miRNAs regulating myogenic differentiation and proliferation (miR-1, miR-206, and miR-133) $[25,26]$. In both groups, we did not observe any significant changes in circulating levels of miR-1 and miR-206. In MUN group, circulating levels of miR-133 transiently decreased within 0.5 hrs, returning to baseline levels $48 \mathrm{hrs}$ after the $10^{\text {th }}$ treatment (Figure 1A, Table 3).

Repeated "Munari" applications specifically modulate miRNAs regulating mitochondrial $\mathrm{Ca}^{2+}$ uptake and pain signaling

Dysregulation of intracellular $\mathrm{Ca}^{2+}$ homeostasis is one of the mechanisms involved in Capsaicin induced pain relief. Therefore, we tested for the expression of miR-25, the negative regulator of MCU channels, responsible for $\mathrm{Ca}^{2+}$ entry into the mitochondria buffering cytosolic $\mathrm{Ca}^{2+}$. In both groups, plasma levels of miR-25 were significantly decreased $48 \mathrm{hrs}$ after the $10^{\text {th }}$ treatment (Figure 1A, Table 3). We also tested the expression of miRNAs 199a and 199b regulating transcription of the TRPV1 receptor, responsible for pain sensation and specific target of Capsaicin. In MUN group, only miR-199a levels significantly decreased only $48 \mathrm{hrs}$ after the $10^{\text {th }}$ treatment (Figure 
1A, Table 3), while in COMB group, both miR-199a and miR-199b significantly increased $0.5 \mathrm{hrs}$ (miR-199b) and $48 \mathrm{hrs}$ (miR-199a) after the treatment (Figure 1B, Table 3).

Statistical analysis by 2 -way ANOVA showed no time $\times$ group interactions, except for miRNA 199a $\left(\mathrm{F}=4.505 ; \mathrm{p}=0.019 ; \eta^{2}=0.231\right)$ meaning that miRNA 199a serum levels changed differently in the two groups between pre and post-10 points in time at a statistically significant value.

\section{Discussion}

Creams, plasters and patches containing low to very high doses of $\mathrm{CP}$ are commonly used to treat several musculoskeletal dysfunctions, including chronic LBP $[2,13,29]$. A single application of high dose CP patches (Qutenza ${ }^{\otimes}$ containing $8 \%$ Capsaicin) is well accepted by Health care providers or social insurances despite serious local reactions (pain, redness, itching, swelling, burning, dryness, rash or bumps) and systemic side effects (increased blood pressure, runny or stuffy nose, sore throat, cough, nausea or vomiting) $[7,8,29]$. In contrast, low dose CP treatment ("Munari" containing 0.09\% Capsaicin) is not recommended by the 'Federation of Austrian Social Insurance Entities' based only on the speculation of possible side effects [12]. Therefore, the present study aimed to test the cumulative effects of 10 applications of "Munari" for pain relief on chronic unspecific LBP as a monotherapy (MUN) or in combination with NMES and classical massage (COMB).

Our results showed that repeated low dose "Munari" treatments have beneficial analgesic effects, improve function and mobility parameters (Table 2) when combined with NMES and classical massage (COMB), with only a short-term hyperemic response at the site of application, without long lasting or negative side effects. In addition, in MUN group, a positive long-lasting side effect of systolic and diastolic blood pressure was observed, indicating that there might be a possible anti-hypertensive effect due to pain relief. Anyhow, further and more focused studies will be necessary to examine this effect.

\section{Effects on inflammatory cascade}

We tested for the safety and efficacy of 10 times "Munari" treatment analysing circulating levels of the major acute phase response and inflammatory biomarkers. In both groups, no significant changes of the selected biomarkers were observed (Table 1 ), indicating that repeated applications of low dose Capsaicin does not induce epithelial cell or blood vessel damage activating the cascade of inflammatory response. Nonetheless, in COMB group miR-451, a negative regulator of TNF- $\alpha$ and IL-6, was increased in the acute phase, even though not significantly, but significantly
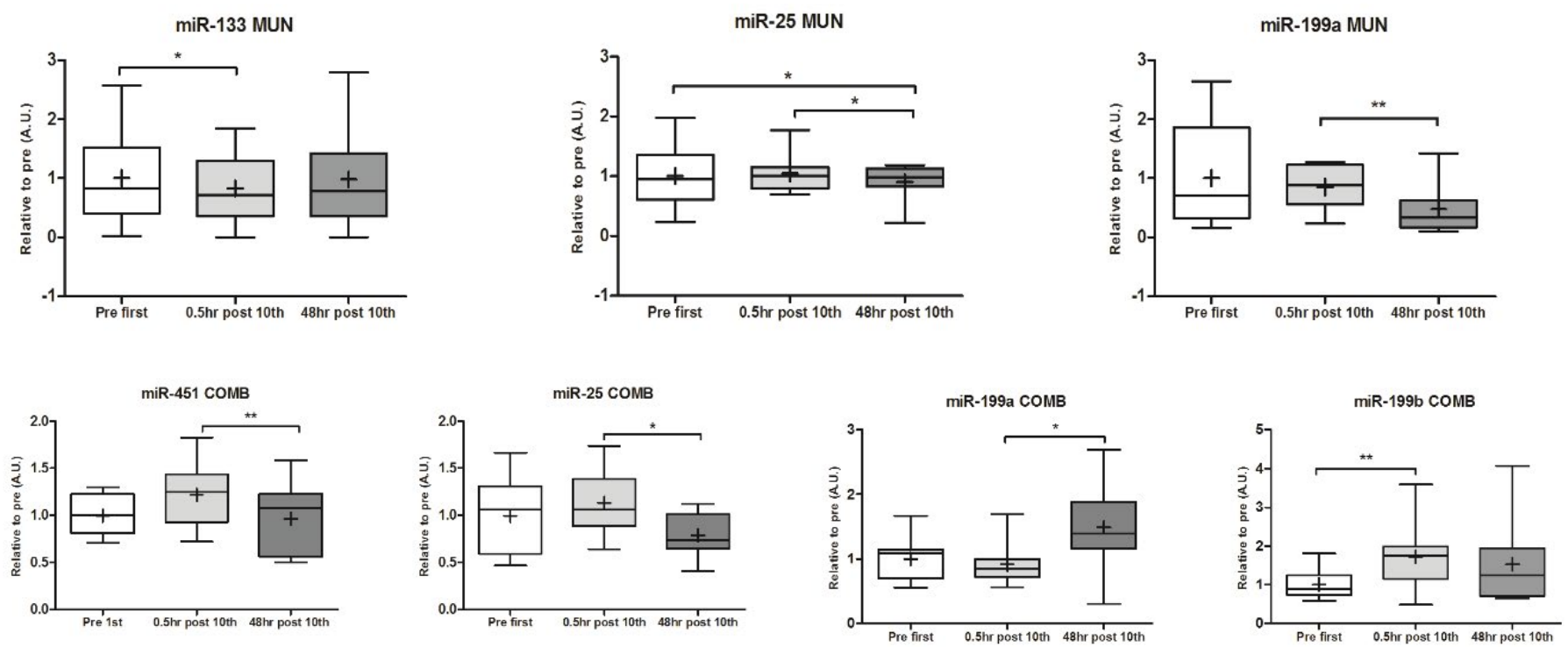

Figure 1A and B: Significant changes of miRNA levels in MUN (A) and COMB (B) induced by the treatment. To baseline miRNA levels before the treatment is assigned a fold change of $1\left(\right.$ Pre $\left.1^{\text {st }}\right)$, to which measurements at different points in time $\left(0.5 \mathrm{hrs}\right.$ and $48 \mathrm{hrs}$ post $\left.10^{\text {th }}\right)$ after the treatment were compared. In all panels, bar and whisker plots are utilized where the horizontal lines denote the statistical median, the crosses denote the statistical mean, and error bars reflect maximum and minimum values. ${ }^{*} \mathrm{p}<0.05 ; * * \mathrm{p}<0.01$

Table 3. miRNAs expression profile significantly dysregulated in LBP subjects after the treatment, related to pain and mobility conditions

\begin{tabular}{|c|c|c|c|c|c|}
\hline & \multicolumn{2}{|c|}{ Time examined } & \multirow[b]{2}{*}{ Target } & \multirow[b]{2}{*}{ Pain } & \multirow[b]{2}{*}{ Mobility } \\
\hline & 0.5 hrs post & 48 hrs post & & & \\
\hline \multirow{3}{*}{ MUN } & - & $\operatorname{miR}-25 \downarrow$ & $\mathrm{MCU}$ & \multirow{3}{*}{ Decreased* } & \multirow{3}{*}{ Unchanged } \\
\hline & - & $\operatorname{miR}-199 \mathrm{a} \downarrow$ & TRPV1 & & \\
\hline & miR-133a $\downarrow$ & - & Skeletal muscle & & \\
\hline \multirow{4}{*}{ COMB } & - & $\operatorname{miR}-451 \downarrow$ & Inflammation & \multirow{4}{*}{ Decreased } & \multirow{4}{*}{ Improved* } \\
\hline & - & 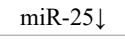 & $\mathrm{MCU}$ & & \\
\hline & - & $\operatorname{miR}-199 \mathrm{a} \uparrow$ & TRPV1 & & \\
\hline & $\operatorname{miR}-199 \mathrm{~b} \uparrow$ & - & TRPV1 & & \\
\hline
\end{tabular}

MCU=Mitochondrial Calcium Uniporter; TRPV1=transient receptor potential cathion channel subfamily V member 1; $\uparrow / \downarrow=$ increase/decrease; *statistically significant changes. 
decreased $48 \mathrm{hrs}$ after the $10^{\text {th }}$ treatment (Figure 1B, Table 3). In has been shown that the miR-451-treatment of mice affected with Rheumatoid Arthritis (SKG mice) reduces the severity of arthritis and the number of infiltrating cells, suppressing neutrophil chemotaxis, thus reducing the inflammatory response [30]. Therefore, the long-term reduction of miR-451 obtained after the COMB therapy may result in a possible anti-inflammatory effect.

A modulation of the neurogenic inflammatory response as assessed by Cortisol and sP-selectin testing was also observed. Significant changes of Cortisol serum levels (catabolic stress hormone, also modulated by inflammation) were detected either in the acute or in the chronic phase response after the $10^{\text {th }}$ application. However, Cortisol serum levels were reduced by the treatment with respect to the pre-values, as a positive long-term effect of the therapy. Furthermore, sP-selectin serum levels were decreased in both groups only in the short-term period $(0.5$ hrs after the application). Since this protein is expressed by activated endothelial cells and platelets during acute inflammatory response, such as the simple vasodilation, the observed transitorial changes may account for the redness of the skin, itching and burning sensations at site of application appearing soon after the application, but that does not persist permanently. In turn, these temporary local site reactions are positive indicators of the effectiveness of the treatment.

In line with these findings, as a sign of the safety of the treatment, no significant changes of the plasma profiles of those miRNAs involved in immuno-inflammatory response and physiological angiogenesis (miR-155, miR-21 and miR-126) [21-23] were observed. Angiogenesis can be activated when the formation of new blood vessels is necessary to restore blood flow in response to injury, therefore the absence of significant changes in circulating levels of those miRNAs regulating angiogenesis may indicate that vessel damage does not occur in the treated areas. Redness, itching and burning sensations are physiological processes activated by Caspaicin binding to specific TRPV1 receptors on epidermal cells, which in turn is an indication of the specificity of the treatment.

\section{Effects on skeletal muscle homeostasis}

MiRNAs expressed in skeletal muscle are modulated during multiple biological processes involved in skeletal muscle homeostasis, in terms of growth, development and maintenance, including atrophy and hypertrophy processes. It is known that miR-1, miR-133 and miR206 play important roles in myogenesis during post-natal growth and in response to injury [26]. Experiments in the C2C12 muscle cell line demonstrated that overexpression of miR-1 or miR-206 promotes myogenic differentiation, while that of miR-133 enhances myoblast proliferation, but represses differentiation [25,26]. After "Munari" treatment, circulating levels of miR-1 and miR-206 were not affected, while only in MUN, miR-133 values decreased only in the acute phase (Figure 1A, Table 3), possibly regulating myoblast proliferation. These results suggest that repeated low dose Capsaicin does not induce skeletal muscle damage, because miRNA pathways regulating muscle myofiber regeneration and differentiation are not long-term activated. Still, miR133 modulation can be a sign of skeletal muscle tissue remodelling, underlying the clinical observation of reduced muscle tension after the treatment.

\section{Effects on pain signaling}

Perturbed $\mathrm{Ca}^{2+}$ homeostasis is one of the possible mechanisms responsible for de-functionalization of afferent pain conducting nerve fibres. It has been shown that miR-25 is a negative regulator of MCU, the channel that drives $\mathrm{Ca}^{2+}$ into the mitochondria. In both MUN and COMB groups, miR-25 plasma levels significantly decreased $48 \mathrm{hrs}$ after the last treatment. The significant reduction of miR-25 plasma levels has a positive effect on MCU transcription and therefore on mitochondrial $\mathrm{Ca}^{2+}$ uptake contributing also to cytosolic $\mathrm{Ca}^{2+}$ buffering. These results show that "Munari" treatment specifically activates signaling pathways regulating intracellular $\mathrm{Ca}^{2+}$ homeostasis, that are underlying mechanism of pain relief [7].

In MUN a significant reduction of miR-199a plasma levels was observed as an acute response, in association to decreased pain. In COMB, miR-199a and miR-199b were both significantly increased, both in the acute or long-term phase. Furthermore, COMB treatment showed increased effects on pain relief and improved mobility. MiR$199 \mathrm{a}$ and miR-119b have been described to be negative regulators of TRPV1 expression [19,20,29]. In vivo up-regulation of miR-199a decreases visceral pain via inhibition of TRPV1 signaling. While in patients with LBS, an inverse correlation between miR-199 a/b and visceral pain scores has been demonstrated [20]. These results suggest that CPC treatment modulates the expression of the specific Capsaicin binding receptor TRPV1, with the consequent reduction of pain and improved mobility, when combined with NMSE and massage (Tables 2 and 3$)$.

\section{Conclusions}

In this study we demonstrated that repeated application of $5 \%$ CP containing $0.09 \%$ Capsaicin, also named as "Munari", is a safe and effective low-cost therapy for the treatment of non-specific chronic LBP inducing pain relief, and specifically modulating miRNAs involved in inflammation and pain signalling. When combined with NMES and classical massage, "Munari" also induces improvement of function and mobility.

Further studies are needed to understand the biological significance and the underlying mechanisms of the observed miRNAs changes. Still, our results indicate miRNAs as promising candidates to better characterize the pathophysiology of pain as well as serological biomarkers to monitor the effects of different therapies in Physical Medicine and Rehabilitation.

\section{Author contributions}

SZ performed miRNA analyses, interpreted results, and wrote the manuscript; NS performed statistical analyses; NS, SL, CH, JC, MS, MK performed enrolment of subjects, functional analyses, collection of demographic data, interpreted the results and contributed to discussion; SL, CH managed, stored and organize shipment of serum and plasma samples; SS, FK, interpreted results and contributed to discussion; WH performed clinical chemistry, and serological analyses of laboratory parameters and inflammatory biomarkers; HK supervised enrolment and functional analyses, interpreted the results, and contributed to discussion.

SZ Sandra Zampieri, NS Nejc Sarabon, SL Stefan Löfler, CH Christian Hofer, SS Sascha Sajer, FK Felix Kabas, JC Jan Cvecka, MS Milan Sedliak, MK Mathias Kern, WH Wolfgang Hübl, HK Helmut Kern.

\section{Acknowledgments}

The authors would like to acknowledge the financial support of the Ludwig Boltzmann Society (Vienna Austria). 


\section{References}

1. Walker BF (2000) The Prevalence of Low Back Pain: A Systematic Review of the Literature from 1966 to 1998. J Spinal Disord 13: 205-207. [Crossref]

2. Frerick H, Keitel W, Kuhn U, Schmidt S, Bredehorst A, et al. (2003) Topical treatmen of chronic low back pain with a capsicum plaster. Pain 106: 59-54. [Crossref]

3. Rubinstein SM, Van Middelkoop M, Kuijpers T, Ostelo R, Verhagen AP, et al. (2010) A systematic review on the effectiveness of complementary and alternative medicine for chronic non-specific low-back pain. Eur Spine J 19: 1213-1238. [Crossref]

4. Paternostro-Sluga T (2017) Rehabilitation peripherer Nervenläsionen. Kompendium Physikalische Medizin und Rehabilitation. pp 411-420.

5. Update der evidenz- und konsensbasierten Österreichischen Leitlinie für das Management akuter, subakuter, chronischer und rezidivierender unspezifischer Kreuzschmerzen. Langfassung 1. Auflage. (2018). Bundesministerium für Arbeit, Soziales, Gesundheit und Konsumentenschutz.

6. Díaz-Franulic I, Caceres-Molina J, Sepulveda RV, Gonzalez-Nilo F, Latorre R (2016) Structure-Driven Pharmacology of Transient Receptor Potential Channel Vanilloid 1. Mol Pharmacol 90: 300-308. [Crossref]

7. Anand P, Bley K (2011) Topical capsaicin for pain management: Therapeutic potential and mechanisms of action of the new high-concentration capsaicin $8 \%$ patch. $\mathrm{Br} J$ Anaesth 107: 490Y502. [Crossref]

8. Baranidharan G, Das S, Bhaskar A (2013) A review of the high-concentration capsaicin patch and experience in its use in the management of neuropathic pain. Ther Adv Neurol Disord 6: 287Y97. [Crossref]

9. Robbins W (2000) Clinical applications of capsaicinoids. Clin J Pain 16: 586-589. [Crossref]

10. Sarabon N, Löfler S, Cvecka J, Hübl W, Zampieri S (2018) Acute effect of differen concentrations of cayenne pepper cataplasm on sensory-motor functions and serum levels of inflammation related biomarkers. Eur J Transl Myol 28: 7333. [Crossref]

11. Grossheim EL (1830) Lehrbuch der operativen Chirurgie. Berlin: Verlag von Theod. Chr. Friedr. Enslin.

12. Enthaler N, Hansbauer B, Sönnichsen A (2012) Die Anwendung, Wirksamkeit und Sicherheit von,,"Munari”"'oder,"“Munari"-Packungen“" in Medizin und Physikalischer Therapie. Paracelsus Medizinische Privatuniversität, Salzburg.

13. Mou J, Paillard F, Turnbull B, Trudeau J, Stoker M, et al. (2014) Qutenza (capsaicin) 8\% patch onset and duration of response and effects of multiple treatments in neuropathic pain patients. Clin J Pain 30: 286-294. [Crossref]

14. Maihofner C, Heskamp ML (2013) Prospective, non- interventional study on the tolerability and analgesic effectiveness over 12 weeks after a single application of capsaicin $8 \%$ cutaneous patch in 1044 patients with peripheral neuropathic pain: First results of the QUEPP study. Curr Med Res Opin 29: 673Y83. [Crossref]
15. Bartel DP (2004) MicroRNAs: genomics, biogenesis, mechanism, and function. Cell 116: 281-297. [Crossref]

16. Atsushi S, Hidenori S (2014) Emerging role of microRNAs in chronic pain Neurochemistry International 77: 58-67. [Crossref]

17. Fairbank JCT, Pynsent PB (2000) The Oswestry Disability Index. Spine 25: 2940-2943. [Crossref]

18. Müller FJ, Schuster C, Weigel B (2013) Klinische Untersuchungstests in Orthopädie und Unfallchirurgie. Springer-Verlag Berlin Heidelberg.

19. Huang Y, Zhang S, Fang X (2018) Plasma miR-199a-5p is increased in neutrophilic phenotype asthma patients and negatively correlated with pulmonary function. PLoS ONE 13: 1-17. [Crossref]

20. Zhou Q, Yang L, Larson S (2016) Decreased miR-199 augments visceral pain in patients with IBS through translational upregulation of TRPV1. Gut 65: 797-805. [Crossref]

21. O'Connell RM, Kahn D, Gibson WS (2010) MicroRNA-155 promotes autoimmune inflammation by enhancing inflammatory T cell development. Immunity 33: 607-609. [Crossref]

22. Sheedy FJ, Palsson-McDermott E, Hennessy EJ, et al. (2010) Negative regulation of TLR4 via targeting of the proinflammatory tumor suppressor PDCD4 by the microRNA miR-21. Nat Immunol 11: 141-147. [Crossref]

23. Olivieri F, Rippo MR, Monsurrò V, Salvioli S, Capri M, et al. (2013) MicroRNAs linking inflamm-aging, cellular senescence and cancer. Ageing Res Rev 12: 1056-1058. [Crossref]

24. Marchi S, Lupini L, Patergnani S, et al. (2013) Downregulation of the mitochondrial calcium uniporter by cancer-related miR-25. Current Biology 23: 58-53. [Crossref]

25. Chen JF, Mandel EM, Thomson JM, et al. (2006) The role of microRNA-1 and microRNA-133 in skeletal muscle proliferation and differentiation. Nat Genet 38: 228 233. [Crossref]

26. Kim HK, Lee YS, Sivaprasad U, Malhotra A, Dutta A(2006) Muscle-specific microRNA miR- 206 promotes muscle differentiation. J Cell Biol 174: 677-687. [Crossref]

27. Tackett MR, Diwan I (2017) Using FirePlex ${ }^{\mathrm{TM}}$ Particle Technology for Multiplex MicroRNA Profiling Without RNA Purification. Methods Mol Biol 1654: 209-219. [Crossref]

28. NeurogesX: Qutenza (2009) (Capsaicin) 8\% Patch Prescribing Information, in Neurogesx, editor. San Mateo, CA, Neurogesx.

29. Sharma SK, Vij AS, Sharma M (2013) Mechanisms and clinical uses of capsaicin. Eur $J$ Pharmacol 720: 55-52. [Crossref]

30. Murata K, Yoshitomi H, Furu M, Ishikawa M, Shibuya H, et al. (2014) MicroRNA-45 down-regulates neutrophil chemotaxis via p38 MAPK. Arthritis Rheumatol 66: 549559. [Crossref]

Copyright: (C2019 Zampieri S. This is an open-access article distributed under the terms of the Creative Commons Attribution License, which permits unrestricted use, distribution, and reproduction in any medium, provided the original author and source are credited. 